\title{
FACTORES ASOCIADOS A ANEMIA EN GESTANTES HOSPITALIZADAS DEL HOSPITAL SAN JOSÉ
}

\author{
Jesús Soto Ramirez ${ }^{1}$
}

\begin{abstract}
RESUMEN
Objetivo: Identificar los factores asociados a la anemia en gestantes hospitalizadas en el servicio de ginecobstetricia del hospital "San José" Callao-Lima. Materiales y métodos: estudio observacional, analítico, transversal con recolección de datos en forma retrospectiva. Resultados: encontramos una prevalencia del $78,9 \%$ de anemia en gestantes. Las gestantes en el 1er trimestre tuvieron mayor porcentaje de anemia $(38,6 \%)(p=0,00) ; 54,6 \%$ de las gestantes menores de 30 años tuvieron anemia ( $p=0,01)$, el odds para la edad fue de 2,2; las gestantes con IMC de 25 a 29,9 fueron las que presentaron mayor porcentaje de anemia $(36,3 \%)(p=0,29)$; las gestantes multíparas fueron las que presentaron mayor anemia $(61,7 \%)$ $(p=0,03)$, el odds para paridad fue de 1,83 ; las gestantes $\sin$ CPN tuvieron mayor porcentaje de anemia $(64,9 \%)(p=0,00)$, el odds calculado fue de 0,03 ; las gestantes que NO presentaron preeclampsia y eclampsia obtuvieron un mayor porcentaje de anemia, $59,1 \%$ y $71,4 \%$ respectivamente. Las gestantes que Si presentaron periodo intergenésico son las que tuvieron mayor anemia $(56,3 \%)(p=0,00)$, el odds calculado fue de 5,52 (IC 95\% $(3,16-9,65))$. Conclusiones: Los factores que se asociaron significativamente a la anemia fueron la edad materna, la edad gestacional, la paridad, los controles prenatales y el periodo intergenésico. Por el contrario, los factores que no se asociaron significativamente fueron la preeclampsia, la eclampsia y el índice de masa corporal.
\end{abstract}

Palabras clave: Anemia Gestacional, embarazo, deficiencia de hierro (Fuente: DeCS BIREME).

\section{FACTORS ASSOCIATED WITH ANEMIA IN HOSPITALIZED PREGNANTS OF THE SAN JOSE HOSPITAL}

\begin{abstract}
Objective: To identify the factors associated with anemia in pregnant women hospitalized in the gynecology and obstetrics service of the "San José" Callao-Lima hospital. Materials and methods: observational, analytical, cross-sectional study with retrospective data collection. Results: we found a prevalence of $78.9 \%$ of anemia in pregnant women. Pregnant women in the 1 st trimester had a higher percentage of anemia $(38.6 \%)(p=0.00) ; 54.6 \%$ of pregnant women under 30 years old had anemia $(p=0.01)$, the odds for age was 2.2; Pregnant women with a BMI of 25 to 29.9 were the ones with the highest percentage of anemia $(36.3 \%)(p=0.29)$; The multiparous pregnant women were the ones that presented greater anemia $(61.7 \%)(p=$ $0.03)$, the odds for parity was 1.83; pregnant women without NPC had a higher percentage of anemia $(64.9 \%)(p=0.00)$, the calculated odds were 0.03 ; pregnant women who did NOT present preeclampsia and eclampsia obtained a higher percentage of anemia, $59.1 \%$ and $71.4 \%$ respectively. The pregnant women who did present an intergenetic period are the ones with the greatest anemia $(56.3 \%)(p=0.00)$, the calculated odds were $5.52(95 \% \mathrm{Cl}(3.16-9.65))$. Conclusions: The factors that were significantly associated with anemia were maternal age, gestational age, parity, prenatal controls and the intergenetic period. In contrast, factors that were not significantly associated were pre-eclampsia, eclampsia, and body mass index.
\end{abstract}

Key words: gestational Anemia, Pregnancy, iron deficiency (Source: MeSH NLM).

\section{INTRODUCCIÓN}

La anemia es la patología hematológica más frecuente del embarazo. Según la OMS, existen 2 billones de mujeres en estado gestacional en el mundo, de las cuales $42 \%$ sufre de algún grado de anemia durante su embarazo ${ }^{1-3}$. En Perú se ha encontrado que 3 de cada 10 gestantes tienen anemia. Según el reporte de ENDES 2016, la prevalencia de gestantes anémicas se elevó al27.9\%, llegando a ser del $38.9 \%$ en las gestantes adolescentes de 15 a 19 años ${ }^{4}$.
El impacto de la anemia en el embarazo puede tener consecuencias severas en la madre cuando esta es severa, sin embargo son leves cuando los niveles de hemoglobina son mayores de $8 \mathrm{mg} / \mathrm{dL}$. Por otro lado, el feto suele ser muy sensible a niveles bajos de hemoglobina menos severos predisponiéndolos a parto prematuro, óbito fetal, bajo peso al nacer, en la vida intrauterina y, anemia infantil, bajo rendimiento escolar, talla baja, trastornos del comportamiento en la vida extrauterina ${ }^{2,5}$.

Médico-Cirujano, Diris Lima Norte, Lima-Perú.

Citar como: Soto J. Factores asociados a anemia en gestantes hospitalizadas del Hospital San José. Rev Peru Investig Matern Perinat 2020; 9 (2): 31-33 DOI https://doi.org/10.33421/inmp.2020203 
Su causa es multifactorial, incluyendo factores nutricionales, sociales, económicos, políticos y ambientales. Tiene como principal etiología la nutricional por deficiencia de hierro, siendo esta responsable de al menos la mitad de los casos de anemia en gestantes. La presente investigación tiene como objetivo conocer los factores asociados a anemia gestacional en la población peruana, lo cual puede servir a futuro a crear planes de tratamiento multidireccionados ${ }^{6,7}$.

\section{MATERIALES Y MÉTODOS}

Se ejecutó un estudio transversal analítico con recolección de datos en forma retrospectiva. La población de estudio estuvo conformada por las gestantes que fueron hospitalizadas en el servicio de Gineco-Obstetricia del Hospital Nacional San José del Callao durante el año 2016. El cálculo del tamaño de la muestra se realizó a partir de una prevalencia esperada del $35 \%$, con un porcentaje de error alfa de $5 \%$ y un nivel de confianza del $95 \%$ para una población infinita. Calculamos un tamaño muestral de 350 sujetos con la calculadora muestral de la Facultad de Medicina, de la Universidad Nacional del Nordeste de Argentina, aplicando un muestreo aleatorio simple de tipo sorteo entre las historias clínicas incluidas en el estudio.

\section{RESULTADOS}

Seleccionamos aleatoriamente 350 gestantes. Encontramos una prevalencia de anemia en gestantes de $78,9 \%$. Nuestra población estuvo conformada un $38,6 \%$ de gestantes en el primer trimestre seguido de las gestantes en el 3er trimestre que representaban el 28,3\% ( $p=0,00)$. En el grupo de las gestantes con anemia, $54,6 \%$ tenían menos de 30 años ( $p$ 0.01 ), el $64,9 \%$ de estas gestantes no tuvieron controles prenatales $(C P N)(p=0,00)$, el $61,7 \%$ fue multípara y $56.3 \%$ tuvieron un periodo intergenésico mayor de 2 años. Con respecto al peso, $36.3 \%$ de las gestantes anémicas tenían sobrepeso (IMC= 25 a $29,9 \mathrm{~kg} / \mathrm{m}^{2}$ ) y $25,4 \%$ de gestantes anémicas sufrían de obesidad ( $\left.\mathrm{IMC}>30 \mathrm{~kg} / \mathrm{m}^{2}\right)$. Las mayoría de las gestantes anémicas no sufrieron preeclampsia $(59,1 \%)$, ni eclampsia $(71.4 \%)$. (tabla1).

El análisis bivariado encontró que las variables de edad [OR de 2,2 (IC 95\% $(1,17-4,48)$ )], controles prenatales [OR 0,03 (IC 95\% $(0,01-0,06))]$, paridad [OR=1,83 (IC 95\% $(1,04-3,21))]$ y periodo intergenésico [OR= $5.42($ IC95\% $(3,16$ - 9,65))] tenían una relación estadísticamente significativa con anemia gestacional. Las variables IMC $(p=0.29)$, preeclampsia $(p=0,72)$, eclampsia $(p=0,72)$ no tuvieron relación con anemia gestacional.

\section{DISCUSIÓN}

El volumen sanguíneo materno en condiciones fisiológicas comienza a aumentar alrededor de las 6 semanas de gestación a expensas del volumen plasmático. La
Tabla 1. Correlación entre los factores de riesgo y la anemia gestacional

\begin{tabular}{lcc}
\hline Factor de riesgo & Frecuencia & $\mathrm{p}$ \\
\hline Anemia gestacional & $78.9 \%$ & \\
Edad gestacional & & \\
$\quad<13 \mathrm{sg}$ & $38.6 \%$ & \\
$14-26 \mathrm{sg}$ & $33.1 \%$ & 0.00 \\
$>27 \mathrm{sg}$ & $28.3 \%$ & \\
Edad materna & & \\
$\quad<30$ años & $54.6 \%$ & 0.01 \\
$>30$ años & $45.4 \%$ & \\
Controles prenatales & & \\
Si & $35.1 \%$ & 0.00 \\
$\quad$ No & $64.9 \%$ & \\
Periodo intergenésico & & \\
Si & $56.3 \%$ & 0.01 \\
$\quad$ No & $43.7 \%$ & \\
Paridad & & \\
Multípara & $61.7 \%$ & 0.00 \\
Paridad & $38.3 \%$ & \\
Peso materno (IMC) & & \\
$\quad<25 \mathrm{~kg} / \mathrm{m}^{2}$ & & \\
$25-29.9 \mathrm{~kg} / \mathrm{m}^{2}$ & $36.3 \%$ & 0.29 \\
$>29.9 \mathrm{~kg} / \mathrm{m}^{2}$ & $25.4 \%$ & \\
Preeclampsia & & \\
Si & $40.9 \%$ & 0.72 \\
No & $59.1 \%$ & \\
Eclampsia & & \\
Si & $28.6 \%$ & \\
No & $71.4 \%$ & \\
\hline
\end{tabular}

Fuente: Ficha de recolección de datos Hospital San José-Callao

hemoglobina materna disminuye progresivamente desde la sexta semana de gestación hasta aproximadamente las 35 semanas, aumentando el mes previo al parto. Su punto más bajo en una paciente sana pero sin suplementación de hierro a $10.5 \mathrm{mg} / \mathrm{dL}^{1,2}$. Según el centro de control y prevención de enfermedades se diagnostica anemia se define anemia a niveles de hemoglobina $(\mathrm{Hb})$ y hematocrito (Hto) menores del quinto percentil de una población de referencia saludable; siendo anemia una $\mathrm{Hb}<11 \mathrm{mg} / \mathrm{dL}$ y $\mathrm{Hto}<33$ en el primer trimestre, una $\mathrm{Hb}<10.5 \mathrm{mg} / \mathrm{dL}$ y $\mathrm{Hto}<32 \%$ en el segundo trimestre y una $\mathrm{Hb} 11 \mathrm{mg} / \mathrm{dL}$ y Hto $33 \%$ en el tercer trimestre ${ }^{2,3}$. Las demandas de hierro durante la gestación aumentan sustancialmente debido al aumento de células rojas y a los requerimientos de la unidad feto placentario por lo que se recomienda una ingesta de $60 \mathrm{mg}$ de hierro elemental diario durante el segundo y tercer trimestre. Sin embargo existen múltiples factores asociados al desarrollo de anemia en este grupo poblacional por lo que es importante reconocerlos en nuestra población 7,8

Nuestros resultados fueron semejantes a diferentes estudios regionales y nacionales ${ }^{2,-9,11-15}$. En Latinoamérica se ha encontrado mayor prevalencia de anemia en el tercer trimestre del embarazo $(56,0 \%)$, observando que la anemia durante el primer trimestre, es un factor 
de riesgo de la anemia al tercer trimestre $(\rho=0,02)^{7}$. En Perú, una investigación en 397 gestantes se encontró una prevalencia general del $35 \%$ de anemia en gestantes, una prevalencia de anemia moderada el $6.3 \%$ y severa del $0.5 \%$, siempre la mayoría gestantes con anemia leve; y al relacionar edad materna y la anemia en gestantes se encontró que existía asociación entre las variables siendo significativamente estadístico, concordando así que la edad materna actúa como factores de riesgo para que la gestante presente anemia ${ }^{10}$.

(18) En estudios de adolescentes embarazadas, el nivel de prevalencia de anemia ferropénica fue de 21,84 $\%$., siendo el factor predisponente en ellas el escaso control prenatal, 9 . Así mismo se ha evidenciado que la multiparidad era más frecuente en la población anémica en un $(69,9 \%)(p<0.05)$ concordando así que la paridad actúa como factores de riesgo para que la gestante presente anemia. De las gestantes evaluadas en una investigación nacional, solo el $10 \%$ desarrollo trastorno hipertensivo (preeclampsia y eclampsia) el 10\% mostrando una $p=$ 0,92 lo cual demuestra que no hay asociación estadística significativa ${ }^{13-15}$. Así mismo con respecto al periodo intergenésico, un investigación peruana encontró que, en 102 gestantes, existe relación significativa entre el intervalo intergenésico corto y la anemia gestacional; siendo en pacientes con intervalo intergenésico corto $(n=102)$ el $94.1 \%$ de las gestantes, presentaron intervalo intergenésico corto entre $12-24$ meses, el $5.9 \%$ un intervalo < 12 meses, de las cuales el $51.0 \%$ presentaron anemia gestacional y el $49 \%$ no presento anemia. ${ }^{9-12}$

Es importante recalcar que las causas de la anemia no son solo nutricionales. La anemia puede ser un marcador de factores sociales y/o ambientales también, por lo que el tratamiento nutricional de por sí solo no necesariamente resuelve el problema ${ }^{15}$.

En conclusión, la edad, la paridad, la presencia de controles prenatales y el periodo intergenésico mayor de 2 años son factores asociados a anemia gestacional. Encontramos una prevalencia alarmante de anemia en gestantes del $78.9 \%$ que implica un problema muy importante que requiere una rápida acción. Se sugieren iniciar intervenciones para concientizar a la población en riesgo de tomar medidas que eviten el desarrollo de anemia durante su gestación.

Financiamiento: Autofinanciado.

Conflicto de interés: El autor declara no tener algún conflicto de interés.

\section{REFERENCIAS BIBLIOGRÁFICAS}

1. New S, Wirth M. Anaemia, pregnancy, and maternal mortality: the problem with globally standardised haemoglobin cutoffs. BJOG Int J Obstet Gynaecol. 1 de enero de 2015;122(2):166-9.
2. Percy L, Mansour D, Fraser I. Iron deficiency and iron deficiency anaemia in women. Best Pract Res Clin Obstet Gynaecol. 1 de abril de 2017;40:55-67.

3. Benoist B de, McLean E, Egll I, Cogswell M. Worldwide prevalence of anaemia 1993-2005: WHO global database on anaemia Worldw Preval Anaemia 1993-2005 WHO Glob Database Anaemia [Internet]. 2008 [citado 13 de julio de 2017]; Disponible en: https://www.cabdirect.org/cabdirect/ abstract/20093013528

4. Munares-García O, GómezGuizado G. Niveles de hemoglobina y anemia en gestantes adolescentes atendidas en establecimientos del Ministerio de Salud del Perú, 2009-2012. Rev Peru Med Exp Salud Publica. julio de 2014;31(3):501-8.

5. Balarajan Y, Ramakrishnan U, Özaltin E, Shankar AH, Subramanian S. Anaemia in lowincome and middle-income countries. The Lancet. 17 de diciembre de 2011;378(9809):2123-35.

6. Breymann C. Iron Deficiency Anemia in Pregnancy. Semin Hematol. 1 de octubre de 2015;52(4):339-47.

7. Barooti E, Rezazadehkermani M, Sadeghirad B, Motaghipisheh S, Tayeri S, Arabi M, et al. Prevalence of Iron Deficiency Anemia among Iranian Pregnant Women; a Systematic Review and Metaanalysis. J Reprod Infertil. 2010;11(1):17-24.

8. Gil Suárez CIS, Villazán Martín C, Ortega San Gil Y. Caracterización de la anemia durante el embarazo y algunos factores de riesgo asociados, en gestantes del municipio regla. Rev Cuba Med Gen Integral. marzo de 2014;30(1):71-81.

9. Ramos Pérez MY, Mejías Álvarez NM, Cardoso Núñez O, Betancourt Nápoles R. Repercusión de la edad materna avanzada sobre el embarazo, el parto y el recién nacido. Rev Arch Méd Camagüey. diciembre de 2006;10(6):56-65.

10. Munares-García O, GómezGuizado G. Anemia en gestantes añosas atendidas en los establecimientos del Ministerio de Salud del Perú, 2009-2012. Rev Peru Epidemiol. 2014;18(2):1-7.

11. Chang S, Zeng L, Brouwer ID, Kok FJ, Yan H. Effect of Iron Deficiency Anemia in Pregnancy on Child Mental Development in Rural China. Pediatrics. 1 de marzo de 2013;131(3):e755-63.

12. Moll R, Davis B. Iron, vitamin B12 and folate. Medicine (Baltimore). 1 de abril de 2017;45(4):198-203.

13. Lopez A, Cacoub P, Macdougall IC, Peyrin-Biroulet L. Iron deficiency anaemia. The Lancet. 27 de febrero de 2016;387(10021):907- 16.

14. Camaschella C. Iron-Deficiency Anemia. N Engl J Med. 7 de mayo de 2015;372(19):1832-43.

15. Mujica-Coopman MF, Brito A, López de Romaña D, Ríos-Castillo I, Cori H, Olivares M. Prevalence of Anemia in Latin America and the Caribbean. Food Nutr Bull. 1 de junio de 2015;36(2_suppl):S119- 28.

\section{Correspondencia:}

Jesús Soto Ramirez

Dirección: Av. Tantamayo N¹200 Urb. Vipol de Naranjal San Martín de Porres

Teléfono: 992200350 